\section{Simple Western Blotting}

R. Westermeier

Freising, Deutschland

Synonym(e) Simple Western

Englischer Begriff Simple Western blotting

Definition Patentierte Methode der Immundetektion von Antigenen nach elektrophoretischer Trennung und Immobilisierung der Proteinfraktionen in Kapillaren.

Physikalisch-chemisches Prinzip Beim Simple Western Blotting erfolgt die Trennung des Proteingemisches mittels einer Kapillarelektrophorese, bei der die innere Kapillarwand mit einer Substanz beschichtet ist, die bei Lichtaktivierung die getrennten Proteinfraktionen bindet. Anschließend folgt eine Immundetektion mit Primär- und Sekundärantikörpern durch Inkubieren und Waschen der Kapillare. Die Sekundärantikörper sind mit Meerrettichperoxidase konjugiert; die Detektion erfolgt über Chemilumineszenz. Alle Schritte sind automatisiert. Die Trennung kann nach 2 verschiedenen Prinzipien durchgeführt werden: entweder durch eine isoelektrische Fokussierung zur Trennung nach den isoelektrischen Punkten oder eine SDS-Elektrophorese zur Trennung nach Molekülgrößen.

Einsatzgebiet Proteinbestimmungen.
Untersuchungsmaterial Biologische Flüssigkeiten, Gewebeextrakte, Zelllysate.

Instrumentalisierung Vollautomatisierte Apparatur der Firma ProteinSimple.

Spezifizität Hoch, da die Methode auf Immunreaktion beruht.

Sensitivität Im Bereich niedriger Picogramm bis hoher Femtogramm.

Fehlermöglichkeit Diese Technik funktioniert vollautomatisch, der Aufwand für die Probenvorbereitung ist minimal. Dadurch wenig Fehler möglich.

Praktikabilität - Automatisierung - Kosten Gerät und Kapillarkits gibt es nur bei der Firma ProteinSimple, der Inhaberin der Patente.

Bewertung - Methodenhierarchie (allg.) Geeignet für klinisch-chemische Labore mit hohem Probendurchsatz.

\section{Literatur}

Nguyen U, Squaglia N, Boge A, Fung PA (2011) The Simple Western ${ }^{\mathrm{TM}}$ : a gel-free, blot-free, hands-free Western blotting reinvention. Nat Methods 8:v-vi 